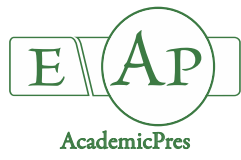

\title{
Physiological Responses and Tolerance Evaluation of Five Poplar Varieties to Waterlogging
}

\author{
Zhongcheng ZHOU ${ }^{1,2 a}$, Gang $\mathrm{LI}^{3,4 \mathrm{~b}}$, Xiaomei SUN ${ }^{1}$, Feng XU ${ }^{3,4 *}$, \\ Zexiong $\mathrm{CHEN}^{5}$ \\ ${ }^{1}$ Chinese Academy of Forestry, Key Laboratory of Tree Breeding and Cultivation of the State Forestry Administration, Research Institute of
Forestry, Beijing 100091, China; $1806725680 @ q q . c o m ; 1628172947 @ q q . c o m$
${ }^{2}$ Hubei Ecology Polytechnic College, Department of Forestry Ecology,Wuhan 430070, China;
${ }^{3}$ Yangtze University, College of Horticulture and Gardening, Jingzhou 434025, Hubei,
China; 15090754721@163.com; xufeng198@126.com ('corresponding author)
Jingzhou 434025, Hubei, China
${ }^{5}$ Chongtze University, Engineering Research Center of Ecology and Agricultural Use of Wetland of Ministry of Education,
Jiversity of Arts and Sciences, Research Institute for Special Plants, Chongqing 402160, China; chenzexiong1979@163.com
}

${ }^{\mathrm{ab}}$ These authors contributed equally to this study

\begin{abstract}
Waterlogging resistance of five poplar varieties, 'Danhongyang' (DHY), 'Juba-261' (JB-261), 'Zongqiansanhao' (ZQ-3), 'Zhonglin-2025' (ZL-2025), and 'Nanlin-895' (NL-895), was evaluated under the simulated waterlogging conditions. Data on changes in leaf color and morphology as well as in biochemical indices, such as chlorophyll, malonaldehyde, soluble protein, soluble sugar content, superoxide dismutase (SOD), peroxidases (POD), ascorbate peroxidase (APX), glutathione reductase (GR), and glutathione peroxidase (GSH-PX) activities, relevant to submergence stress, were analyzed. The principal component analysis of the data identified the waterlogging resistance coefficient of the indices, which showed that waterflooding brought about different degrees of damage in the five poplar varieties, with DHY having the lowest waterlogging index. The leaf pigment content of the poplar was remarkably decreased by waterlogging, whereas malondialdehyde (MDA) and proline contents were enhanced, but in different extents among the poplar varieties. Nearly all other poplar varieties showed a tendency of decline in JB-261, ZQ-3, ZL-2025, NL-895, except for SOD activity in DHY, which increased under submergence stress. Poplar varieties had varying degree of changes in POD activity, and APX activity tended to increase upon waterlogging. GR also displayed increasing tendency in JB-261, ZL-2025 and NL-895, except for in ZQ-3, which declined under waterlogging stress. GSH-PX except for ZQ-3 displayed no significant change, which showed a tendency of decline in DHY, JB-261, ZL-2025, and NL895. Principal component analysis allowed us to reduce16 indices to four independent indices. The subordinate function analysis identified that the DHY variety had the highest waterlogging tolerance, whereas the NL-895 variety had the lowest waterlogging tolerance among tested varieties.
\end{abstract}

Keywords: evaluation of comprehensive; physiological response differences; poplar; varieties; waterlogging resistance

\section{Introduction}

Waterlogging is the second major agricultural disaster in China, accounting for approximately $24 \%$ of natural disasters (Wang et al., 2015). Excessive soil moisture disrupts the water balance of plants and has a remarkable impact on plant morphology and metabolism, thereby restricting plant growth (Thomas et al., 2003; Ye et al., 2003). Submergence deprives plants of oxygen and inhibits aerobic respiration, resulting in the accumulation of toxic substances such as ethanol and lactic acid. Thus, absorption of minerals was inhibited, changed in the hormonal level of plants and disordered metabolic (Visser et al., 1994; Geigenberger, 2003; Kaelke and Dawson, 2003). In the mung bean under submergence stress, the activities of superoxide dismutase (SOD), peroxidase, glutathione reductase, and ascorbate peroxidase (APX) are elevated at the beginning of flooding to eliminate free radicals accumulating in the cells (Ahmed et al., 2002). Upon flood treatment, the primary root of Camptotheca acuminata 
gradually rots; root activity declines, lactate dehydrogenase activity increases, and the $\mathrm{O}_{2}^{-}, \mathrm{H}_{2} \mathrm{O}_{2}$, and malonaldehyde contents of the leaf gradually increase. Submergence stress induces increase of the antioxidant enzyme activity in various plant cells in varying degrees, explaining the different waterlogging tolerance of plants to some extent (Keles and Öncel, 2002).

The Jianghan Plain in China is located in the middle and lower reaches of the Yangtze River. It has large areas of low wetlands and mudflats. The presence of vast wetland and aquatic plant species provide an opportunity for the development and study of waterlogging-resistant tree species. Because annual precipitation in Jianghan Plain has been increasing year by year recently, the demand for waterlogged poplar varieties has also been increasing. In spite of the great number of research and fruitful achievements on directive breeding, genetics, and seed breeding, systematic research on the waterlogging resistance of poplar, especially on its physiological and biochemical aspects, is still limited. As a result, the selection of waterlogging-resistant poplar varieties suitable for Jianghan Plain is still in the initial stage. Therefore, the present experiment was conducted to illustrate the response mechanism of different poplar varieties against submergence stress so as to establish a waterlogging evaluation system of poplar in Jianghan Plain.

\section{Materials and Methods}

\section{Plant materials and treatments}

The experimental site was located in the botanical garden of Yangtze University in Jingzhou, Hubei, China (around N30.35, E112.14). The experimental materials were 1-year old poplar cuttings taken from five cultivars: 'Danhongyang' (DHY), 'Juba-261' (JB-261), 'Zhongqian-3' (QZ-3), 'Zhonglin-2025' (ZL-2025) and 'Nanlin-895' (NL-895). Planting of the cuttings was started on March 20,2017 . The stem cuttings were planted in plastic flowerpots $(10 \mathrm{~cm}$ in inner diameter and $25 \mathrm{~cm}$ in depth). The culture medium was vermiculite, coconut shell powder and pearl, uniformly mixed with the ratio of 5:3:2 and placed in the greenhouse for seedling cultivation, two cutting seedlings of each pot. During this period, the cultivation and management measures were undertaken to maintain consistency until the end of the flooding. The cutting seedlings that reached at uniform growth of about $80 \mathrm{~cm}$ height were selected for waterlogging treatment on July 17 . The experiment was designed as a random group with 3 replications, and every cultivar was treated with 9 pots, three plants per pets. The flooding treatment was such that the water surface was about $10 \mathrm{~cm}$ above the soil surface, and water was replenished daily to keep the water level constant. Control plants were watered every evening to maintain the soil moisture content at $60-80 \%$ of the maximum field water capacity.

During this period of the experiment, the morphological characteristics were recorded every 10 days with photo record. After flooding for 65 days, three mature leaves in the middle section of the plants were harvested and immediately frozen in liquid nitrogen to store at $-80{ }^{\circ} \mathrm{C}$. The frozen materials were used to determine their physiological and biochemical indices.
Index determination

The degree of harm due to waterlogging was classified at the end of flood as follows: Grade 1: leaves were naturally outward and all green; Grade 2: yellow or curly individual leaves; Grade 3: about 1/5 of the leaves show yellowing, the leaves lose water and wither and droop; Grade 4: about 2/5 leaves scorched; Grade 5: more than 3/5 leaves scorched and dry, fallen leaves or dead branches; Grade 6: above 4/5 or more leaves are scorched and dry, almost all leaves fall off or branches died.

Injury index was obtained by the following equation.

Injury index $=$ (injury grade $\times$ injury number of plants) $/$ (Total number of plants $\times$ Waterlogging maximum)

After 65 days of submergence treatment, the roots, stems, and leaves of the plants were separately harvested and their wet and dry biomass were measured.

Chlorophyll a, chlorophyll b, and total chlorophyll content were determined according to the method of Wellburn (1994). Chlorophyll content [mg/g FW (fresh weight)] was calculated at 665,649 , and $470 \mathrm{~nm}$ by spectrophotometer (UV-2800, Unico, GER).

Soluble sugar content was determined using anthrone colorimetric method (Moustakas et al., 2011). Fresh tissue samples (5 g) were collected and ground into a homogeneous slurry. Then, the ground samples were extracted with $6 \mathrm{ml}$ of sulfosalicylic acid for $2 \mathrm{~h}$, filtered by a funnel, and centrifuged at $4,000 \times \mathrm{g}$ for $5 \mathrm{~min}$. The supernatant after centrifugation was used to calculate the soluble sugar content $[\mathrm{mg} / \mathrm{g} \mathrm{FW}$ (fresh weight)] at a wavelength of $630 \mathrm{~nm}$ by a spectrophotometer.

The MDA content was extracted using thiobarbituric acid (TBA) reagent and boiled at $100{ }^{\circ} \mathrm{C}$ for $20 \mathrm{~min}$ as previously described by Yang (2009). After cooling to room temperature and centrifugation at 3,000 rpm for $10 \mathrm{~min}$, the MDA concentrations were determined at 450, 532 and $600 \mathrm{~nm}$ of absorbance with a spectrometer. The MDA concentration can be estimated through the formula $\mathrm{C}$ $(\mu \mathrm{mol} / \mathrm{l})=6.45\left(\mathrm{~A}_{532}-\mathrm{A}_{600}\right)-0.56 \mathrm{~A}_{450}$. The MDA concentration was expressed as $\mu \mathrm{mol} / \mathrm{g}$ FW. (FW, fresh weight)

Proline content was estimated using described method with L-proline as standard (Yang et al., 2011). Briefly, the plant samples $(0.5 \mathrm{~g})$ were homogenized in $5 \mathrm{ml}$ of $3 \%$ sulphosalicyclic acid solution. After centrifugation, $2 \mathrm{ml}$ supernatant, $2 \mathrm{ml}$ glacial acetic acid and $2 \mathrm{ml} \mathrm{2.5 \%}$ acid ninhydrin solution were added into a test tube covered with Teflon cap. Well mixed solutions were boiled at $100{ }^{\circ} \mathrm{C}$ for $40 \mathrm{~min}$. After cooling to room temperature, the proline level of samples was calculated at $520 \mathrm{~nm}$ absorbance by making the specification curve with known concentration of Lproline. The proline concentration was expressed as $\mu \mathrm{g} / \mathrm{g}$ FW.

The activities of antioxidant enzymes (T-SOD, CuZn SOD, POD, APX, Total-Protein, GR, and GSH-PX) were determined according to the instructions of corresponding kits (NanjingJiancheng Biotech Co., Nanjing, China).

\section{Statistical analysis}

Calculation of waterlogging resistance coefficient (Zhu et al., 2017) was as follows. Waterlogging tolerant coefficient $=[$ Process measurement $/$ Control test value $] \times$ $100 \%(1)$. 
660

Principal component analysis of waterlogging tolerant was performed on the waterlogging tolerant of individual photosynthetic indexes. The original single indicator was transformed into a new independent comprehensive indicator.

The membership function value of the composite index was obtained by the formula of Zhou et al. (2003). $U\left(X_{j}\right)=$ $\left(X_{j}-X \min \right) /(X \max -X \min ), j=1,2, \ldots .$. , where, $X_{j}$ represents the Jth comprehensive index, Xmin represents the Jth minimum comprehensive index value, Xmax represents the Jth maximum comprehensive index value. If a certain index is negatively correlated with waterlogging tolerance, the value of its waterlogging tolerance membership function can be calculated through the inverse membership function.

The specific formula is as follows: $\mathrm{X}(\mu)=1-(\mathrm{X}-\mathrm{Xmin}) /$ (Xmax-Xmin). The comprehensive value of waterlogging tolerance of varieties was obtained by summing up the specific waterlogging subordinate values of each index. The higher the comprehensive value was, the stronger the waterlogging resistance.

The weight of each comprehensive index was calculated according to the contribution rate of the comprehensive index (Rui-Juan et al., 2013). $\mathrm{W}_{\mathrm{j}}=\mathrm{P}_{\mathrm{j}} / \Sigma(\mathrm{j}=1)^{\wedge} \mathrm{nP}_{\mathrm{j}}=1,2, \ldots \ldots$. Where, $W_{j}$ value represents the importance of the Jth comprehensive index in all comprehensive indexes, and $\mathrm{P}_{\mathrm{j}}$ is the contribution rate of the Jth comprehensive index of each variety.

Comprehensive assessment was determined as follows:

$$
\mathrm{D}=\Sigma_{\mathrm{j}}=\left[\mathrm{U}\left(\mathrm{X}_{\mathrm{j}}\right) \times \mathrm{W}_{\mathrm{j}}\right], \mathrm{j}=1,2, \ldots \ldots
$$

Data were analyzed with one-way ANOVA using SPSS22.0 for Windows (SPSS Inc., Chicago, IL). The means were compared with Duncan's multiple range tests. $P$ $<0.05$ was considered to be statistically significant.

\section{Results}

Effect of waterlogging stress on the growth of poplar varieties

Biomass change is an important index of plant resistance. Once the five poplar varieties experienced submergence stress, their biomass change displayed remarkable differences among them (Table 1). During the submergence treatment for $65 \mathrm{~d}$, the biomass of 'Danhongyang' (DHY) increased by $140 \%$ compared with that before submergence, indicating that DHY can grow well even under submergence condition. However, the biomass of the (JB-261, QZ-3, ZL-2025 and NL-895 varieties was increased by only 15 to $45 \%$ indicating that they were severely affected by waterlogging stress. On the basis of biomass change, the waterlogging tolerance of DHY was judged to be higher than those of the four other varieties.

\section{Effect of waterlogging stress on the leaf morphology}

The changes in the leaves and root system in the five poplar varieties after submergence for $65 \mathrm{~d}$ were as follows. Decaying and yellowing in the large area of the leaves, as well as margin rolling, leaf abscission, were observed in four poplar varieties, namely, ZO-3, JB-261, ZL-2025, and NL895 . Only a small area of yellowing is found in the leaf at the lowest level (Fig. 1), and emerald is still relatively high as a whole, especially for ZL-2025, which has a waterlogging injury index as high as 0.69 (Table 2), thereby showing poor waterlogging resistance. Nevertheless, no large-scale yellowing and rolling were found in the DHY leaf, whose waterlogging injury index was only 0.28 , thereby showing strong waterlogging resistance.

The stem bases of all the five poplar varieties underwent submergence treatment showed varying degree of adventitious root appearance.

Effect of waterlogging stress on the content of leaf pigment, soluble sugar, and soluble protein of the poplar varieties.

Fig. 2 shows that the contents of chlorophyll a, chlorophyll $\mathrm{b}$, carotenoid and the total chlorophylls of five poplar varieties under waterlogging treatment were remarkably lower than those of the corresponding control group, albeit with different degrees among varieties. It is of note that decreases in contents of the total chlorophyll and carotene of JB-261 under submergence treatment was lower compared to control values, and the chlorophyll a, chlorophyll b, carotenoid and the total chlorophylls are all $60-80 \%$ of the control group.

Table 1. Effects of waterlogging on biomass of poplar clones

\begin{tabular}{|c|c|c|c|c|c|c|c|c|c|}
\hline \multirow{2}{*}{ Cultivar } & \multicolumn{4}{|c|}{ July 17} & \multicolumn{4}{|c|}{ October 2} & \multirow{2}{*}{$\begin{array}{c}\text { Biomass } \\
\text { percent }(\%)\end{array}$} \\
\hline & Frw & Fsw & Flw & Ttfw & Frw & Fsw & $\mathrm{Fl}_{\mathrm{w}}$ & $\mathrm{Ttfw}$ & \\
\hline DHY & $8.75 \pm 0.36$ & $15.23 \pm 0.58$ & $7.89 \pm 0.20$ & $31.88 \pm 0.62$ & $13.43 \pm 0.12$ & $49.31 \pm 0.30$ & $12.82 \pm 0.12$ & $75.54 \pm 0.15$ & 140 \\
\hline ZQ-3 & $12.82 \pm 0.31$ & $20.94 \pm 0.41$ & $9.14 \pm 0.47$ & $42.61 \pm 0.21$ & $13.55 \pm 0.09$ & $19.47 \pm 0.31$ & $19.47 \pm 0.72$ & $61.38 \pm 0.78$ & 44 \\
\hline JB-261 & $15.33 \pm 0.48$ & $16.61 \pm 0.25$ & $9.83 \pm 0.11$ & $41.75 \pm 0.44$ & $11.27 \pm 0.32$ & $26.11 \pm 0.46$ & $26.11 \pm 0.19$ & $52.14 \pm 0.61$ & 25 \\
\hline ZL-2025 & $18.34 \pm 0.68$ & $32.34 \pm 0.43$ & $20.92 \pm 0.34$ & $71.61 \pm 0.39$ & $25.96 \pm 0.59$ & $38.71 \pm .0 .13$ & $30.64 \pm 0.18$ & $95.31 \pm 0.51$ & 33 \\
\hline NL-895 & $15.49 \pm 0.59$ & $11.92 \pm 0.34$ & $17.77 \pm 0.62$ & $45.18 \pm 0.32$ & $16.15 \pm 0.09$ & $16.52 \pm 0.15$ & $19.79 \pm 0.20$ & $52.46 \pm 0.25$ & 16 \\
\hline
\end{tabular}

Frw, Fsw, Flw and Ttfw represent Fresh root weight, Fresh stem weight, Fresh leaf weight, the total fresh weight.

Table 2. Leaf injury index by waterlogging of five poplar varieties

\begin{tabular}{ccc}
\hline Cultivar & Injury index \\
\hline DHY & 0.28 & 0.54 \\
ZQ-3 & 0.50 \\
JB-261 & 0.69 \\
ZL-2025 & 0.62 \\
NL-895 & & 0.62 \\
\hline
\end{tabular}




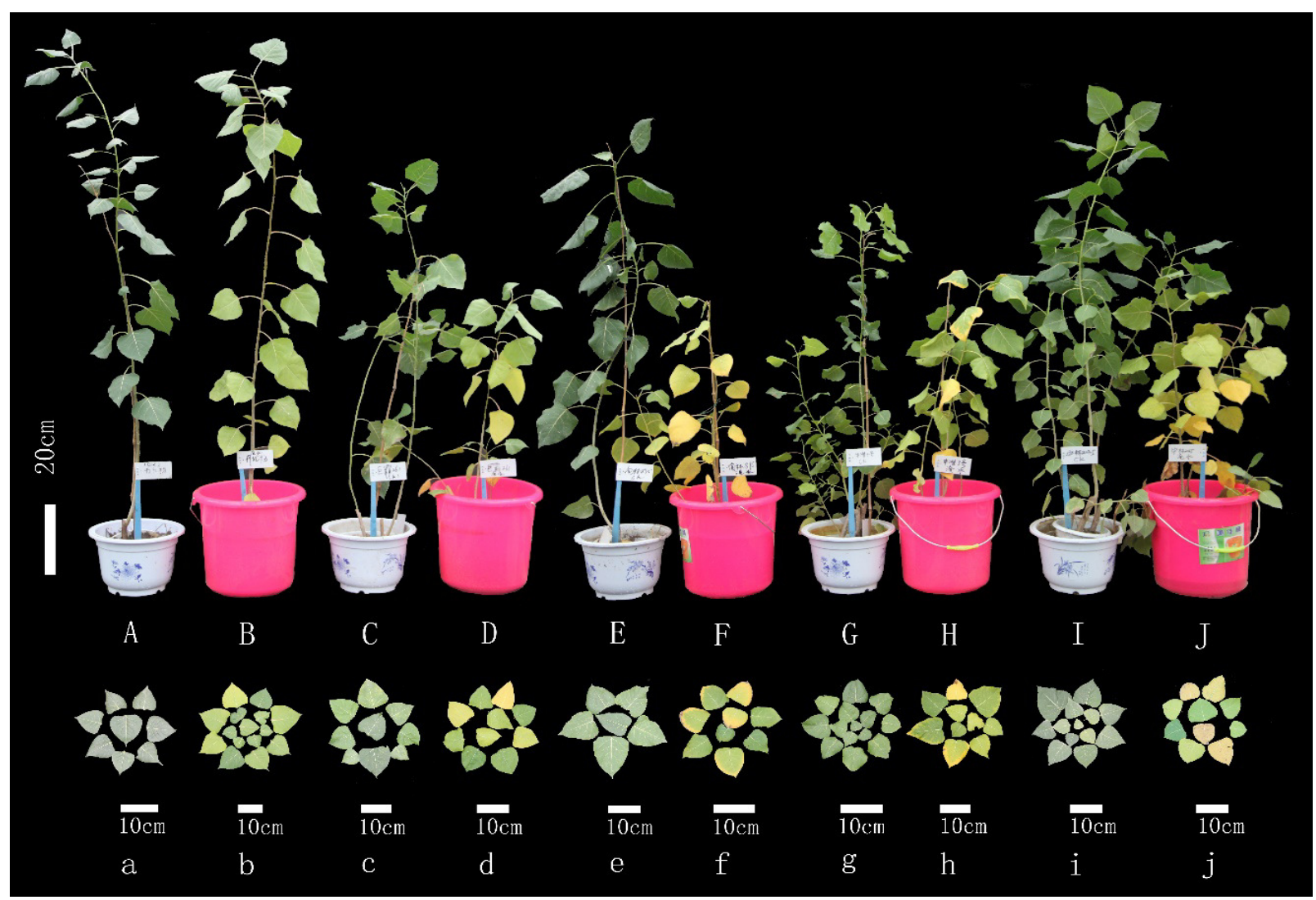

Fig. 1. Comparison of the morphology of the leaves of five poplar varieties under submergence and control after the submergence stress of 65d. The external morphology of DHY (A/a, B/b), JB-261 (C/c, D/d), NL895 (E/e, F/f), ZQ-3 (G/g, H/h) and ZL$2025(\mathrm{I} / \mathrm{i}, \mathrm{K} / \mathrm{k}$ ) under normal growth condition (white pot)/submergence treatment (red pot). The scale bars of the whole poplar tree are $20 \mathrm{~cm}$, and the scale bars of leaves is $10 \mathrm{~cm}$

However, ZL-2025 and NL-895 exhibited most severe decrease in chlorophyll a, chlorophyll b, carotenoid, and the total content of chlorophyll contents. The values were all lower by approximately $35 \%$ than that of the control group. The four pigment indices of 'Zongqiansanhao' (ZQ-3) under submergence stress remained at approximately $50 \%$ of the control group. These results showed that DHY and JB-26 under submergence condition could keep their leaf pigment content at a high level. Hence, the analysis confirmed the stronger waterlogging resistance of DHY and JB-26 than other poplar varieties. In addition, chlorophyll $a$ / chlorophyll $b$ (chl $a / \mathrm{chl} b$ ) was no significant changes in DHY and ZQ-3 of control compared to waterlogging, suggesting that it was slight resistance under waterlogging stress. However, chl $a / \mathrm{chl} b$ appeared to be an increasing trend in the JB-261 and ZL-2025 of control compared to waterlogging and decreasing tendency in the NL-895 of control compared to waterlogging (Fig. 2E).

Submergence for $65 \mathrm{~d}$ caused no significant changes in soluble sugar content in the leaves of ZL-2025 and NL-895 (Fig. 3A). At the same time, soluble sugar in the leaves of the other three varieties exhibited considerable increase, $25 \%$ for DHY, 70\% for ZQ-3, and 65\% for JB-261 compared to that of the control group.

Submergence treatment had different impacts on the soluble protein content among the poplar varieties. The three other poplar varieties all had remarkable increases in
ZQ-3, JB-261 and NL-895, compared with the control group, in which DHY and ZL-2025 had large increasing amplitudes. The soluble protein in the leaf in DHY and ZL 2025 increased by $80 \%$ and $94 \%$ compared with the control group, respectively.

Effect of submergence stress on the content of malonaldehyde and proline in the leaf of poplar varieties

Fig. 4A shows that MDA content in the ZL-2025 leaf was $41 \%$ higher than that of the control group. In contrast, MDA content in DHY, JB-261 and NL-895 were lower than the control group by $26 \%, 28 \%$ and $24 \%$. ZQ-3 did not show remarkable change in MDA content. The proline content increase was highest in the leaves of DHY up to 49\%, and in JB-261, ZL-2025 and NL-895 were higher than the control group by $38 \%, 33 \%$ and $33 \%$. (Fig. $4 \mathrm{~B}$ ). However, proline content of ZQ-3 had no significant difference compared with the control group.

\section{Effect of submergence stress on antioxidant enzyme activity} in the leaf of poplar varieties

Submergence stress can greatly affect SOD enzyme activity (Fig. 5A) in the leaves of poplar trees, but with different degrees of influence. Under submergence stress, $T$ SOD and CuZn SOD activities in the leaf of only the DHY variety were higher by 45 and $118 \%$ than those of the control group, respectively (Fig. 5A and B). 
662
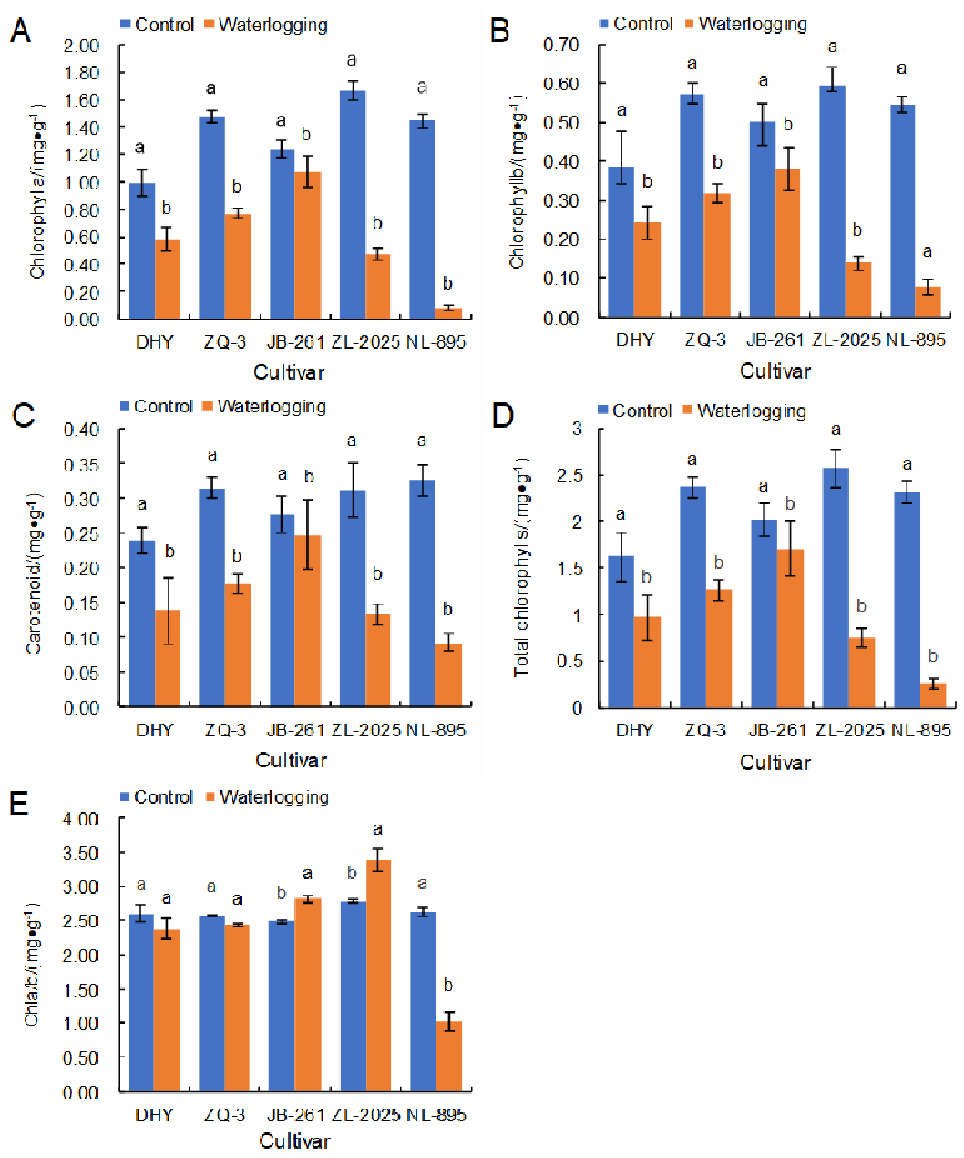

Fig. 2. The effect of waterlogging on chlorophyll a (A), chlorophyll b (B), carotenoid (C), total chlorophylls content (D) and the ratio chl $\mathrm{a} / \mathrm{chl} \mathrm{b}(\mathrm{E})$ of leaves of poplar. Means and standard errors are shown from three biological replicates, and different letters on the same columns indicate significant differences at $\mathrm{P}<0.05$
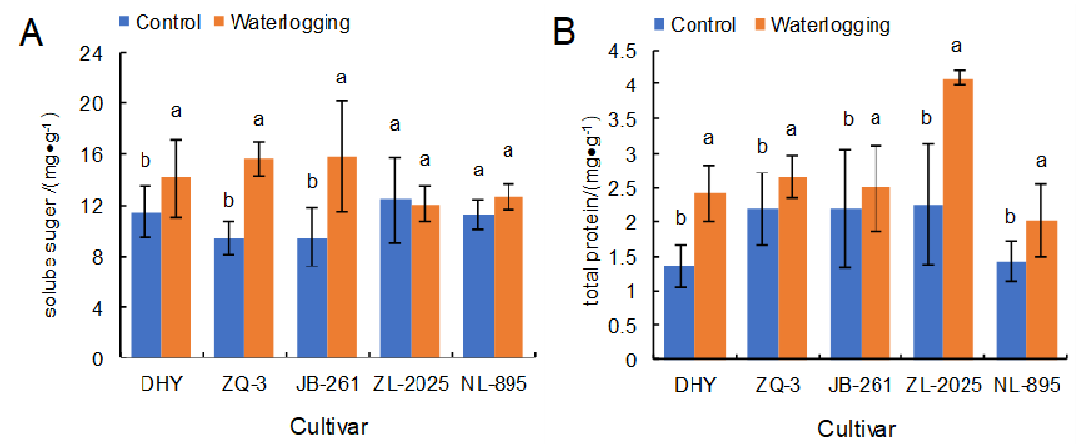

Fig. 3. The effect of waterlogging on soluble sugar (A) and total protein content (B) of leaves of poplar. Means and standard errors are shown from three biological replicates, and different letters in the same columns indicate significant differences at $P<$ 0.05

Unlike the DHY variety, T-SOD and CuZn SOD activities of the four other poplar varieties were decreased significantly, most notably in ZQ-3 for T-SOD and JB-261 and NL-895 for CuZn SOD. This result showed that submergence stress resulted in different responses of SOD activities in the leaves of different poplar varieties. Positive increase of both T-SOD and CuZn SOD activities in the DHY suggested that this variety possessed high waterlogging tolerance by inducing SOD to cope with the submergence stress.
Fig. 5C shows the changes in POD activities in the varieties. DHY and JB-261 showed significant increase of SOD activity by 110 and $200 \%$, respectively, whereas ZQ-3 and ZL-2015 experienced decrease in the activity. NL-895 was intermediate with small increase of POD activity in $16 \%$. Induction of the POD activity upon submergence of poplar is an important defense mechanism (Yiu et al., 2009). The data well explains adaptability of DHY, JB-261, and NL-895 against the submergence stress. 

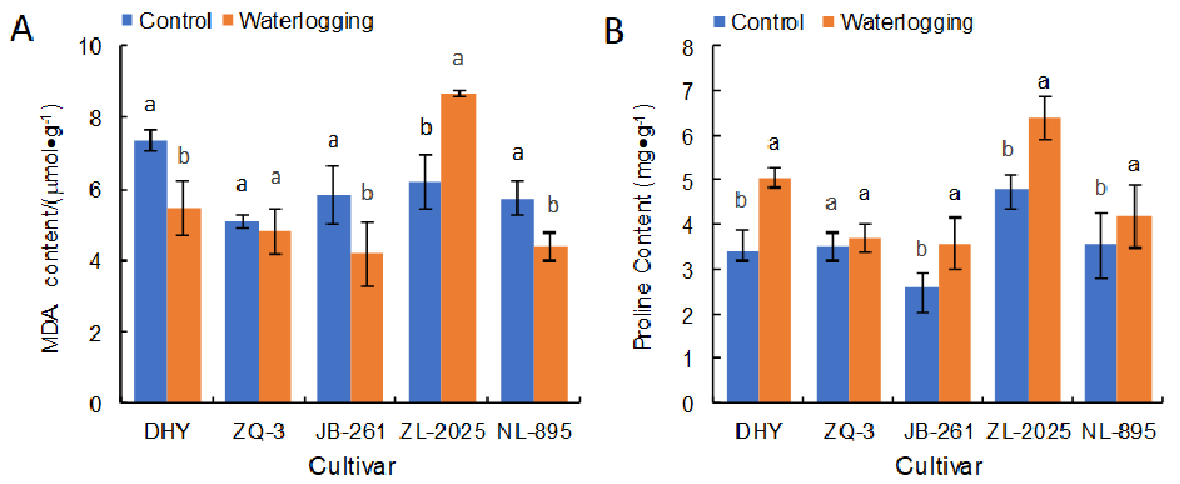

Fig. 4. The effect of waterlogging on MDA content (A) and proline content (B) of leaves of poplar. Means and standard errors are shown from three biological replications, and different letters in the same columns indicate significant differences at $\mathrm{P}<0.05$.
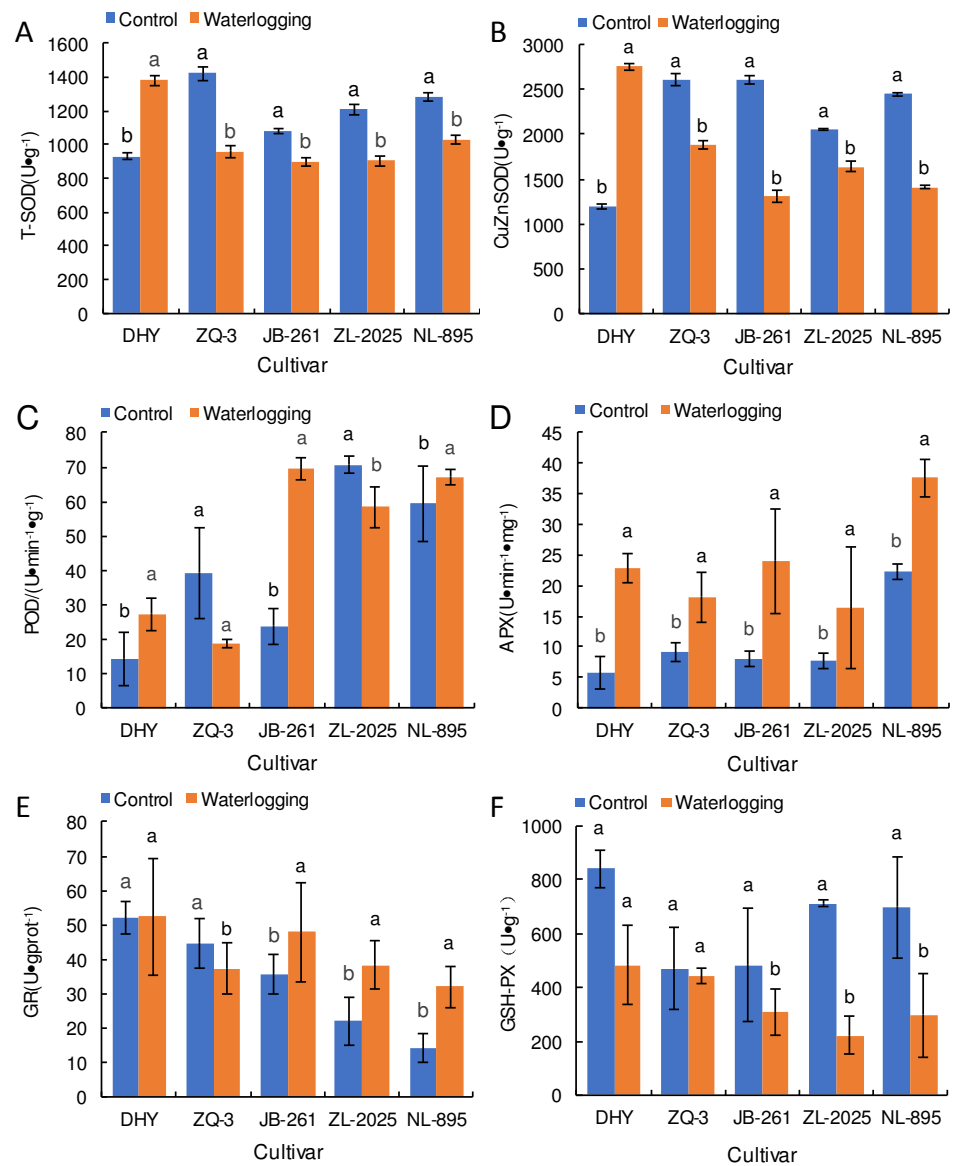

Fig. 5. The effect of waterlogging on T-SOD (A), CuZnSOD (B), POD (C), APX (D), GR(E) and GSH-PX (F) activity in the leaf of five poplar cultivars. Means and standard errors are shown from three biological replicates, and different letters in the same columns indicate significant differences at $\mathrm{P}<0.05$.

When subjected to submergence stress for $65 \mathrm{~d}$, ascorbate peroxidase (APX) activity in the leaf of all the five poplar varieties was remarkably induced (Fig. 5D). DHY and JB-261 were 345 and $195 \%$ higher than that of the control group $(\mathrm{P}<0.05)$, respectively. The remaining three other poplar varieties, ZQ-3, ZL-2025, and NL-895, also showed significant yet lower than those of the other two ranging 103 to $68 \%$ increase. This outcome again reconfirmed the high water-logging tolerance of DHY and JB-261.

Submergence caused little change in GR activity for DHY and increase in the activity for JB-261, ZL-2025, and
NL-895, ranging from 40 to $155 \%$ (Fig. 5E). Even though ZQ-3 showed decrease in GR activity, the extent was only $17 \%$ (Fig. 5E). Therefore, change in GR activity did not explain water-logging tolerance among the varieties. It is possible that preexisting high GR activity in DHY did not call for further induction of GR.

The effect of submergence on GSH-PX activity in the five poplar varieties again did not follow the pattern of water-logging stress (Fig. 5F). GSH-PX activities in four poplar varieties were significantly lowered upon waterlogging stress by 31 to $69 \%$, except for ZQ-3 which showed no significant difference. 
664

Comprehensive analysis and evaluation of poplar waterlogging resistance

The principal component analysis was performed based on the related waterlogging-resistance coefficients of morphological and biological indices of the five poplar varieties (Table 5).

It can be seen from the correlation coefficient matrix (Table 3) that all physiological indicators have positive and negative correlations to different extent, so that the information they provided was overlapped. The contribution rate of the first four comprehensive indicators CI (1)-CI (4) was 0.474, 0.307, 0.134, and 0. 084. If the sum of the four indicators reaches 1.0, the contribution of all indicators is fully integrated. This contribution transferred the 16 original single indices to four new and relatively independent comprehensive indices, which completely represent the information content of the 16 indices. At the same time, the relative importance of each comprehensive index can be estimated according to the size of the contribution rate. The comprehensive index value of each poplar was calculated according to the index coefficient of each comprehensive index (Table 5) and the waterlogging resistance coefficient of each single index (Tables 3 and 6).

The weight of four comprehensive indices can be calculated according to the CI contribution rate of each principal component, namely, CI (1)-CI (4), as given above. The $\mathrm{D}$ value of the comprehensive waterlogging resistance of poplar varieties was calculated according to the comprehensive evaluation formula (Table 6), in which the maximum $\mathrm{D}$ value of $\mathrm{DHY}$ is 0.671 . The minimum $\mathrm{D}$ value of NL-895 is 0.082 . The waterlogging resistance of the five poplar varieties was ranked according to the $D$ value: DHY > JB-261 > ZQ-3>ZL2025>NL-895. The results showed that DHY poplar had the highest waterlogging tolerance, whereas NL-895 poplar had the lowest tolerance.

Table 3. The value of waterlogging resistant coefficients of each physiological index

\begin{tabular}{ccccccccccccccccc}
\hline Variety & \multirow{2}{*}{ Sugar } & chl a & chl b & Car & T-chls & $\begin{array}{c}\text { GSH- } \\
\text { PX }\end{array}$ & Protein & MDA & POD & GR & APX & Pro & CuSOD & TSOD & FwoB & DwoB \\
\hline DHY & 1.25 & 0.58 & 0.64 & 0.58 & 0.6 & 0.57 & 1.8 & 0.74 & 2.11 & 1 & 4.45 & 1.49 & 2.18 & 1.45 & 1.03 & 1.01 \\
ZQ-3 & 1.7 & 0.52 & 0.56 & 0.56 & 0.53 & 0.8 & 1.24 & 0.94 & 0.52 & 0.8 & 1.98 & 1.07 & 0.76 & 0.64 & 0.8 & 0.77 \\
JB-261 & 1.65 & 0.88 & 0.78 & 0.91 & 0.86 & 0.69 & 1.36 & 0.72 & 3 & 1.4 & 2.95 & 1.38 & 0.67 & 0.79 & 0.46 & 0.45 \\
ZL-2025 & 1.05 & 0.28 & 0.23 & 0.44 & 0.29 & 0.31 & 1.97 & 1.41 & 0.83 & 1.9 & 2.03 & 1.33 & 0.74 & 0.75 & 0.9 & 0.89 \\
NL-895 & 1.13 & 0.05 & 0.22 & 0.28 & 0.11 & 0.4 & 1.41 & 0.76 & 1.16 & 2.6 & 1.68 & 1.33 & 0.64 & 0.81 & 0.69 & 0.88 \\
\hline
\end{tabular}

${ }^{*}$ The correlation significant at $\mathrm{P}=0.05,{ }^{* *}$ at $\mathrm{P}=0.01$

Table 4. Correlation matrix of each physiological index

\begin{tabular}{|c|c|c|c|c|c|c|c|c|c|c|c|c|c|c|c|c|}
\hline Index & Sugar & $\mathrm{Chl} \mathrm{a}$ & Chl b & Car & Tchls & GSHPX & Protein & $\mathrm{MDA}$ & POD & GR & APX & Pro & CuSOD & TSOD & FW & DW \\
\hline Sugar & 1.000 & & & & & & & & & & & & & & & \\
\hline $\mathrm{Chl} \mathrm{a}$ & 0.740 & 1.000 & & & & & & & & & & & & & & \\
\hline Chl b & 0.771 & 0.957 & 1.000 & & & & & & & & & & & & & \\
\hline Car & 0.717 & $0.977^{*}$ & 0.904 & 1.000 & & & & & & & & & & & & \\
\hline Tchls & 0.743 & $0.999^{* *}$ & 0.969 & $0.972^{*}$ & 1.000 & & & & & & & & & & & \\
\hline GSHPX & 0.960 & 0.714 & 0.806 & 0.637 & 0.726 & 1.000 & & & & & & & & & & \\
\hline Protein & -0.762 & -0.221 & -0.345 & -0.252 & -0.237 & -0.711 & 1.000 & & & & & & & & & \\
\hline MDA & -0.420 & -0.347 & -0.573 & -0.326 & -0.388 & -0.512 & 0.592 & 1.000 & & & & & & & & \\
\hline POD & 0.263 & 0.707 & 0.714 & 0.759 & 0.720 & 0.230 & -0.050 & -0.593 & 1.000 & & & & & & & \\
\hline GR & -0.662 & -0.733 & -0.762 & -0.582 & -0.736 & -0.779 & 0.123 & 0.150 & -0.156 & 1.000 & & & & & & \\
\hline APX & 0.056 & 0.572 & 0.651 & 0.457 & 0.592 & 0.226 & 0.338 & -0.416 & 0.643 & -0.562 & 1.000 & & & & & \\
\hline Pro & -0.495 & 0.127 & 0.137 & 0.145 & 0.141 & -0.439 & 0.578 & -0.247 & 0.682 & 0.196 & 0.683 & 1.000 & & & & \\
\hline CuSOD & -0.181 & 0.220 & 0.348 & 0.056 & 0.244 & 0.063 & 0.451 & -0.287 & 0.281 & -0.485 & 0.901 & 0.574 & 1.000 & & & \\
\hline TSOD & -0.295 & 0.169 & 0.301 & 0.046 & 0.196 & -0.076 & 0.471 & -0.379 & 0.427 & -0.282 & 0.903 & 0.755 & 0.963 & 1.000 & & \\
\hline $\mathrm{FW}$ & -0.500 & -0.323 & -0.279 & -0.489 & -0.320 & -0.286 & 0.666 & 0.367 & -0.449 & -0.261 & 0.361 & 0.139 & 0.691 & 0.566 & 1.000 & \\
\hline DW & -0.707 & -0.644 & -0.546 & -0.772 & -0.632 & -0.499 & 0.581 & 0.253 & -0.524 & 0.109 & 0.166 & 0.182 & 0.563 & 0.515 & $0.906^{*}$ & 1.000 \\
\hline
\end{tabular}

${ }^{*}$ The correlation significant at $\mathrm{P}=0.05,{ }^{* *}$ at $\mathrm{P}=0.01$.

Table 5. Coefficients of comprehensive indexes $[\mathrm{CI}(\mathrm{x})]$ and proportion $(\mathrm{P})$

\begin{tabular}{|c|c|c|c|c|c|c|c|c|c|c|c|c|c|c|c|c|c|}
\hline Index & Sugar & $\mathrm{Chl} \mathrm{a}$ & $\mathrm{Chl} \mathrm{b}$ & Car & Tchls & GSHPX & Protein & MDA & POD & GR & APX & Pro & CuSOD & TSOD & $\mathrm{FW}$ & DW & $P$ \\
\hline $\mathrm{CI}(1)$ & 0.304 & 0.347 & 0.357 & 0.339 & 0.351 & 0.301 & -0.17 & -0.2 & 0.259 & -0.251 & 0.188 & 0.02 & 0.066 & 0.053 & -0.159 & -0.247 & 0.474 \\
\hline $\mathrm{CI}(2)$ & -0.202 & 0.044 & 0.073 & -0.008 & 0.051 & -0.114 & 0.313 & -0.1 & 0.147 & -0.106 & 0.386 & 0.349 & 0.422 & 0.433 & 0.308 & 0.268 & 0.307 \\
\hline $\mathrm{CI}(3)$ & 0.208 & 0.005 & 0.047 & -0.112 & 0.005 & 0.311 & -0.07 & 0.15 & -0.42 & -0.453 & 0.008 & -0.43 & 0.172 & -0.01 & 0.395 & 0.259 & 0.134 \\
\hline $\mathrm{CI}(4)$ & -0.056 & 0.241 & 0.002 & 0.276 & 0.204 & -0.175 & 0.471 & 0.67 & -0.01 & -0.148 & -0.02 & 0.01 & -0.144 & -0.2 & 0.073 & -0.172 & 0.084 \\
\hline
\end{tabular}

Table 6. The value of comprehensive index $[\mathrm{CI}(\mathrm{x})]$, index weight $(\mathrm{IW}), \mathrm{U}(\mathrm{x}), \mathrm{Y}(\mathrm{x})$ and $\mathrm{D}$ of each variety

\begin{tabular}{ccccccccccccccc}
\hline Variety & $\mathrm{CI}(1)$ & $\mathrm{CI}(2)$ & $\mathrm{CI}(3)$ & $\mathrm{CI}(4)$ & $\mathrm{U}(1)$ & $\mathrm{U}(2)$ & $\mathrm{U}(3)$ & $\mathrm{U}(4)$ & $\mathrm{Y}(1)$ & $\mathrm{Y}(2)$ & $\mathrm{Y}(3)$ & $\mathrm{Y}(4)$ & $\mathrm{D}($ value $)$ \\
\hline DHY & 0.880 & 3.730 & 0.450 & -0.310 & 0.574 & 1.000 & 0.528 & 0.250 & 0.272 & 0.307 & 0.071 & 0.021 & 0.671 \\
ZQ-3 & 0.890 & -1.970 & 2.150 & -0.050 & 0.576 & 0.000 & 1.000 & 0.337 & 0.273 & 0.000 & 0.135 & 0.028 & 0.436 \\
JB-261 & 3.630 & -0.980 & -1.450 & 0.250 & 1.000 & 0.174 & 0.000 & 0.437 & 0.474 & 0.053 & 0.000 & 0.037 & 0.564 \\
ZL-2025 & -2.830 & 0.140 & -0.230 & 1.940 & 0.000 & 0.370 & 0.339 & 1.000 & 0.000 & 0.114 & 0.046 & 0.084 & 0.243 \\
NL-895 & -2.580 & -0.940 & -1.230 & -1.060 & 0.039 & 0.181 & 0.061 & 0.000 & 0.018 & 0.055 & 0.008 & 0.000 & 0.082 \\
IW & & & & & 0.474 & 0.307 & 0.135 & 0.084 & & \\
\hline
\end{tabular}




\section{Discussion}

Submergence stress inhibits plant growth from many aspects. However, under submergence condition, plants with waterlogging tolerance can resist the damages brought by environmental changes by transforming their Physiology signs and morphologies (Bailey-Serres and Voesene, 2008). Submergence stress changes chlorophyll content and Composition and destroys photosynthetic mechanism and performance by slowing down the growth of leaves and roots, thus inhibiting the growth and biomass accumulation of plants (Voesenek et al., 2013; Kissmann et al., 2014). The degree of growth inhibition varied with poplar clones. Under submergence stress, the biomass of DHY could still maintain growth, albeit in a lower rate compared with that without submergence, showing strong waterlogging resistance. However, JB-261 was the most affected, with its roots severely inhibited by waterlogged stress, and showed poor waterlogging resistance.

Many studies showed that when plants suffer from submergence stress, chlorophyll a, chlorophyll b, and total chlorophyll contents in the leaf of the plants decrease. Ge et al. (2014) showed that water stress decreases not only chlorophyll (Zhou et al., 2017) but also carotenoid content. In the present study, chlorophyll and carotenoid content both displayed a decreasing tendency compared with the control group. The decreasing tendencies of chlorophyll and carotenoid in the leaves of DHY and JB-261 were lower compared with those of other poplar varieties, indicating DHY and JB-261 had stronger waterlogging tolerance than other poplar varieties.

Soil flooding exposes plant roots to hypoxia or anoxia, which severely limits availability of energy and sugar, and produces toxic substances such as ethyl alcohol and acetaldehyde (Sairam et al., 2008), severely affecting plant growth. High levels of soluble substances, such as soluble sugar and protein (Yin et al., 2009), accumulate in plants to ensure adjustment toward osmotic stress. Pociecha (2013) posited that plants could improve their waterlogging resistance and survival under submergence stress by accumulating additional carbohydrate stores. Kreuzwieser $e t$ al. (2015) assumed that some sensitive plants consume soluble sugar after being submerged for a while, whereas some waterlogging-resistant plants maintain a stable and adequate supply of carbohydrates in the process of flooding to regulate glycolysis, which is the key for woody plants to survive under hypoxic stress. In the present experiment, the soluble sugar content of DHY, ZQ-3, and JB-261, all showing high waterlogging resistance, still remained at a high level. However, other factors need to be combined to determine which variety has the strongest waterlogging tolerance. Soluble protein content in plants affects its growth development and aging process. Under waterlogging condition, the variety with strong waterlogging resistance has more soluble protein contents than the variety with weak waterlogging resistance (Lorenzo et al., 1995). Although soluble protein content of all the five poplar varieties were higher than that of the control group, DHY and ZL-2025 showed the highest fold increase in protein content $(P<0.01)$ compared with that of the control group, suggesting that DHY and ZL-2025 had the most outstanding waterlogging resistance among the five poplar varieties.

MDA is the final product of membrane lipid peroxidation. The protective ability of the tissues is weakened when MDA accumulation is high. Generally, the degree of membrane lipid peroxidation in the poplar varieties with high flood-resistance is lower than the varieties with low flood-resistance (Loreti et al., 2010). In the present study, the MDA content of the tested poplar varieties, with exception of ZL-2025, were decreased compared with that of the control group. Especially, DHY, JB-261N, and NL-895 showed significant decrease. This finding indicates that these three poplar varieties had an ability to prevent membrane lipid peroxidation and thus explains high resistance of the varieties against waterlogging. Proline accumulation in the plant leaf is usually caused by osmotic stress (Zhu et al., 2009). In the present experiment, the observed significant increase in proline content of DHY under waterlogging condition could account for the high resistance of DHY against water stress.

To resist the toxicity of reactive oxygen species under submergence stress, plants also have developed complex antioxidant defense systems. Primary defense employs nonenzyme substances, such as ascorbic acid, glutathione, and vitamin $\mathrm{E}$ and further defense is achieved by enzymes such as active-oxygen-processed enzymes (SOD and POD), APX, GR, glutathione peroxidase (GSH-PX), as well as the antioxidant regeneration enzymes. Combined force of these systems can alleviate the damage of reactive oxygen species to plants under submergence stress (Thirunavukkarasu et al., 2009). Kumutha et al. (2009) showed that the antioxidant enzyme activity of the waterlogging-resistant genotype of cajanus increased continuously when exposed to the submergence condition, whereas the antioxidant enzyme activity of the sensitive genotype decreased after submergence for $2 \mathrm{~d}$. High SOD activity is known to be important for plants to resist submergence stress (Tan et al., 2009). In the present experiment, T-SOD and CuZn SOD activities in the DHY variety under submergence stress increased significantly, thereby showing strong resistance toward oxidative stress. The POD activities of DHY, JB261, and NL895 were remarkably higher than that of the control group (Fig. 5C). The high POD activities of the varieties can explain considerable waterlogging resistance of these poplar cultivars, as Zhu (2017) had demonstrated with peony. Although the APX activities of all the tested poplar varieties were considerably higher than that of the control group (Fig. 5D), DHY exhibited the highest fold increase (about 300\% increase compared to control) among the varieties. This again indicates high resistance of DHY against waterlogging.

GSH-PX, together with SOD, peroxide (POD), and catalase (CAT), constitutes the major cellular antioxidant enzyme system (Blokhina et al., 2003; Islam and Macdonald, 2004). GSH-PX mainly removes the lipid and hydrogen peroxides, and thus protects the macromolecules of the biological membrane from being destroyed by Oxidation. Ascorbic acid-glutathione cycle, where GR plays an important role in maintaining intracellular ASA and GSH levels, is an important means to detoxify reactive oxygen in plants (Shu et al., 2011). The present experimental result showed that the GR activities in the 
666

four other poplar varieties are either negatively (ZQ-3) or positively (JB-261, ZL-2025, and NL-895) affected by the submergence stress, except for DHY whose activity remained virtually unchanged by water stress (Fig. 5E). Submergence stress lowered GSH-PX activities in all the tested poplar varieties, but in different degrees. The activities in DHY and ZQ-3 were least affected, indicating higher waterlogging tolerance.

Waterlogging-resistant woody plants can adapt to flooding through morphological adaptation and physiological regulation (Shimamura et al., 2010; Argusa $e t$ al., 2015; Loreti et al., 2016). Therefore, waterlogging resistance is a compound trait, including morphological growth, physiology, biochemistry, and molecular expression (Sairam et al., 2008). A single index is not accurate enough to reflect the waterlogging resistance of plants. Thus, multiple indices should be used to evaluate the waterlogging resistance of plants (Wang et al., 2011). In this study, changes in morphology characteristics, growth status, and physiological and biochemical indices of plants were referred to assess the waterlogging tolerance of five poplar varieties in conjunction with principals component analysis. Multi-index method was used to measure the waterlogging tolerance of the five poplar varieties. In this study, the waterlogging tolerance of various poplar varieties was comprehensively evaluated by using the methods of principal component analysis of membership function as follows: DHY>JB-261>ZQ-3>ZL2025>NL-895. This result is basically consistent with the observed result of the morphology. Of the five poplar varieties, DHY had the highest waterlogging tolerance, whereas NL-895 the lowest tolerance. In the present study, the waterlogging resistant germplasm selection and waterlogging tolerance evaluation system for poplar varieties were established in terms of the characteristics of the response mechanism to waterlogging stress. The results provide a theoretical basis for exploring the mechanism of waterlogging resistance and selecting varieties with high waterlogging resistance, thereby establishing a reference for breeding poplar with waterlogging resistance. In order to further verify poplar varieties strong waterlogging resistance, further studies will be carried out, and transcriptome data will be analyzed in the follow-up to study the specific reasons for the expression of different waterlogging resistance genes in different varieties from the transcriptional level, so as to provide theoretical basis for their stress tolerance breeding.

\section{Conclusions}

Our results have been obtained by determining multiple physiological indexes which have closely related to the waterlogging resistance of poplar varieties. Principal component analysis of five poplar varieties was carried out; through the confirmation of morphology, injury index and physiology, we finally determined that DHY has the strongest waterlogging resistance of the five varieties. Therefore, DHY with strong waterlogging resistance in production can be selected and planted with areas with more rainfall. In areas where there was not much rain, JB261, ZQ-3, ZL2025, and NL-895 varieties with moderate waterlogging resistance can be selected.

\section{Acknowledgements}

This study was supported by the Demonstration Project of Forestry Science and Technology funded by the Central Government of China (The Plan of Forestry Department of Hubei Province, Grant No. [2015]HBTK07) and Hubei Provincial Key Technology Support Program of Forestry (Grant No. Lykjizc201209).

\section{References}

Ahmed S, Nawata E, Hosokawa M, Domae Y, Sakuratani T (2002). Alterations in photosynthesis and some antioxidant enzymatic activities of mungbean subjected to waterloging. Plant Science 163(1):117-123.

Argus RE, Colmer TD, Grierson PF (2015). Early physiological flood tolerance is followed by slow post-flooding root recovery in the dryland riparian tree Eucalyptus camaldulensis subsp. refulgens: Physiological flood tolerance and recovery. Plant Cell and Environment 38(6):11891199.

Bailey-Serres J, Voesenek L (2008). Flooding stress: Acclimations and genetic diversity. Annual Review of Plant Biology 59(59):313.

Blokhina O, Virolainen E, Fagerstedt KV (2003). Antioxidants, oxidative damage and oxygen deprivation stress: a review. Annals of Botany 91(2):179-194.

Geigenberger P (2003). Response of plant metabolism to too little oxygen. Current Opinion in Plant Biology 6(3):247-256.

Ge Y, He X, Wang J, Jiang B, Ye R, Lin X (2014). Physiological and biochemical responses of Phoebe bournei, seedlings to water stress and recovery. Acta Physiologiae Plantarum 36(5):1241-1250.

Islam MA, Macdonald SE (2004). Ecophysiological adaptations of black spruce (Picea mariana) and tamarack (Larix laricina) seedlings to flooding. Trees 18(1):35-42.

Kaelke CM, Dawson JO (2003). Seasonal flooding regimes influence survival, nitrogen fixation, and the partitioning of nitrogen and biomass in Alnusincana ssp.rugosa. Plant and Soil 254(1):167-177.

Keleş Y, Öncel I (2002). Response of antioxidative defence system to temperature and water stress combinations in wheat seedlings. Plant Science 163(4):783-790.

Kissmann C, da Veiga EB, Eichemberg MT, Eichemberg MT, Habermann G (2014). Morphological effects of flooding on Styrax pohlii and the dynamics of physiological responses during flooding and post-flooding conditions. Aquatic Botany 119:7-14.

KreuzwieserJ, RennenbergH (2015). Molecular and physiological responses of trees to waterlogging stress. Plant Cell and Environment 37(10):2245-2259.

Kumutha D, Ezhilmathi K, Sairam RK, Srivastava GC, Deshmukh PS (2009). Waterloging induced oxidative stress and antioxidant activity in pigeonpea genotypes. Biologia Plantarum 53(1):75-84.

Lorenzo G, Pierdomenico P, Amedeo A (1995). Effect of anoxia on carbohydrate metabolism in rice seedlings. Plant Physiology 108(2):735741.

Loreti E, Van VH, Perata P (2016). Plant responses to flooding stress. Current Opinion in Plant Biology 33:6471. 
Moustakas M, Sperdouli I, Kouna T, Antonopoulou CI, Therios I (2011). Exogenous proline induces soluble sugar accumulation and alleviates drought stress effects on photosystem II functioning of Arabidopsis thaliana leaves. Plant Growth Regulation 65(2):315.

Pociecha E (2013). Different physiological reactions at vegetative and generative stage of development of field bean plants exposed to flooding and undergoing recovery. Journal of Agronomy and Crop Science 199(3):195-199.

RuiJM, Zhang BB, CaiZX, ShengZJ, YU ML (2013).Evaluation of peach rootstock waterlogging tolerance based on the responses of the photosynthetic indexes to continuous submergence stress. Acta Horticulturae 31(4):1064-1072.

Sairam RK, Kumutha D, Ezhilmathi K, Deshmukh PS, Srivastava GC (2008). Physiology and biochemistry of waterlogging tolerance in plants. Biologia Plantarum 52(3):401-412.

Shimamura S, Yamamoto R, Nakamura T, Shimada S, Komatsu S (2010). Stem hypertrophic lenticels and secondary aerenchyma enable oxygen transport to roots of soybean in flooded soil. Annals of Botany 106(2):277-284.

Shu DF, Wang LY, Duan M, Deng YS, Meng QW (2011). Antisensemediated depletion of tomato chloroplast glutathione reductase enhances susceptibility to chilling stress. Plant Physiology and Biochemistry 49(10):1228-1237.

Tan SD, Zhu MY,Zhang KR, Dang HS, ZhangQF (2009). Response and adaptation of plants to submergence stress. Chinese Journal of Ecology 28(9):1871-1877.

Thirunavukkarasu N, Hossain F, Mohan S, Shiriga K, Mittal S, Sharma R, ... Gupta H S (2013). Genome-wide expression of transcriptomes and their co-expression pattern in subtropical maize (Zea mays L.) under waterloggingstress. PLoSOne 8(8):e70433.

Thomas AL, Guerreiro SM, Sodek L (2005). Aerenchyma formation and recovery from hypoxia of the flooded root system of nodulated soybean. Annals of Botany 96(7):1191-1198.

Visser EJW, Voesenek LACJ, Vartapetian BB, Jackson MB (1994). Flooding and plant growth. Annals of Botany 91(2):107-109.

Voesenek L, Bailey-Serres J (2013). Flooding tolerance: $\mathrm{O}_{2}$, sensing and survival strategies. Current Opinion in Plant Biology 16(5):647-653.

Wang J (2015). Effect of waterlogging on physiological characteristics of peony. Chinese Journal of Ecology 34(12):3341-3347.

Wang SG, Wang ZL, Wang P, Wang HW, Li, F, Huang W, Yin YP (2011). Evaluation of wheat freezing resistance based on the responses of the physiological indices to low temperature stress. Acta Ecologica Sinica 31(4):1064-1072.
Wellburn AR (1994). The spectral determination of chlorophylls a and b, as well as total carotenoids, using various solvents with spectrophotometers of different resolution. Journal of Plant Physiology 144(3):307-313.

Ye Y, Tam NFY, Wong YS, Lu CY (2003). Growth and physiological responses of two mangrove species (Bruguiera gymnorrbiza, and Kandelia candel) to waterlogging. Environmental and Experimental Botany 49(3):209-221.

Yin D, Chen S, Chen F, Guan Z, Fang W (2009). Morphological and physiological responses of two chrysanthemum cultivars differing in their tolerance to waterlogging. Environmental and Experimental Botany 67(1):87-93.

YangF, Wang Y, Wang J, Deng, WQ, Liao L, Li, M (2011). Different ecophysiological responses between male and female Populus deltoides clones to waterloging stress. Forest Ecology and Management 262(11):19631971.

Yang F, Xu X, Xiao X, Li C (2009). Responses to drought stress in two poplar species originating from different altitudes. Biologia Plantarum 53(3):511-516.

Yiu JC, JuangLD, Fang YT, Liu CW, Wu SJ (2009). Exogenous putrescine reduces flooding-induced oxidative damage by increasing the antioxidant properties of welsh onion. Scientia Horticulturae, 120(3):306-314.

Zhang XZ (1992). The measurement and mechanism of lipid peroxidation and SOD, POD, SOD, POD and CAT activities in biological system. In: Zhang XZ (Ed). Research Methodology of Crop Physiology. Agriculture Press, Beijing,pp 208-211.

Zhou C, Bai T, Wang Y, Wang Y, Wu T, Zhang X, ... Han Z (2017). Morpholoical and enzymatic responses to waterlogging in three Prunus, species. Scientia Horticulturae 221:62-67.

Zhou G, Mei F, Zhou Z, Zhu X (2003). Comprehensive evaluation and forecast on physiological indices of waterlogging resistance of different wheat varieties. Scientia Agricultura Sinica 11:1378-1382.

Zhu H, Zu YG, Wang WJ (2009). Effect of proline on plant growth under different stress conditions. Journal of Northeast Forestry University 37(4):86-89.

Zhu X, Jin S, Jianguo AI, Wang X (2017). Evaluation of waterloging tolerance of peony variety. Journal of Nuclear Agricultural Sciences 31(3):607-613. 